\title{
Knowledge transfer when learning a second programming language
}

\author{
Ioan Alfred Letia \\ Technical University of Cluj-Napoca \\ Romania
}

\begin{abstract}
Student models form a very important issue in research concerning cognitive processes. This paper discusses knowledge transfer which takes place when students learn a second programming language. Reasoning by analogy is used to describe this transfer and the way in which students use previous knowledge in solving new problems. An experimental instrument to study these issues is discussed and also some problems concerning the building of taxonomies for the two programming languages.
\end{abstract}

Main conference themes: artificial intelligence, tutoring

Educational areas:

Study topics: computer literacy

Secondary keywords: knowledge representation 


\section{INTRODUCTION}

One of the main directions of artificial intelligence research is the study of human cognitive processes. An important issue in this research is to establish the information which a teacher should provide students with in order to ensure a successful instructional process [1]. The success of this process may be evaluated through testing of the ability of the students to solve new problems in the particular domain.

While learning a second programming language, students use both conceptual and procedural programming knowledge which they already possess. The facilitation of learning has been researched from the perspective of user interfaces with knowledge bases [2], intelligent tutoring systems with user modelling [3] and human-computer interaction with mental models for enhanced predictive and explanatory powers [4]. The representation of knowledge used by learners while learning is no trivial task $[1,3,4,5,6,7,8]$.

The aim of the paper is to clarify how the increase in pragmatic knowledge of learners of programming languages will develop when moving to a second language. Case based reasoning techniques complemented with reasoning by analogy are used to model the cognitive process which might take place in the mind of the learner. Although the idea of a mental model is at the moment very much debated, a reasonable model is required if we are to assess and help the learning process.

\section{PROGRAMMING LANGUAGE TAXONOMY}

The study of knowledge transfer when learning a second programming language must include a model covering the two programming paradigms in order to understand their common and differing aspects. A taxonomy of the main concepts of a domain can be very useful in the study of human problem solving processes [9]. The main difficulty in creating such a taxonomy in this field is the lack of theoretical models for reasoning about programs. There are some theoretical formalisms for functional program analysis and transformation, but reasoning about procedural programs is a difficult task due to lateral effects.

We consider subsets of two different programming languages: PASCAL and a simple functional language. These subsets ignore some aspects such as input/output instructions as these are irrelevant for our case. Below a taxonomy for the main concepts relevant to the programming process in a functional programming language is shown. 


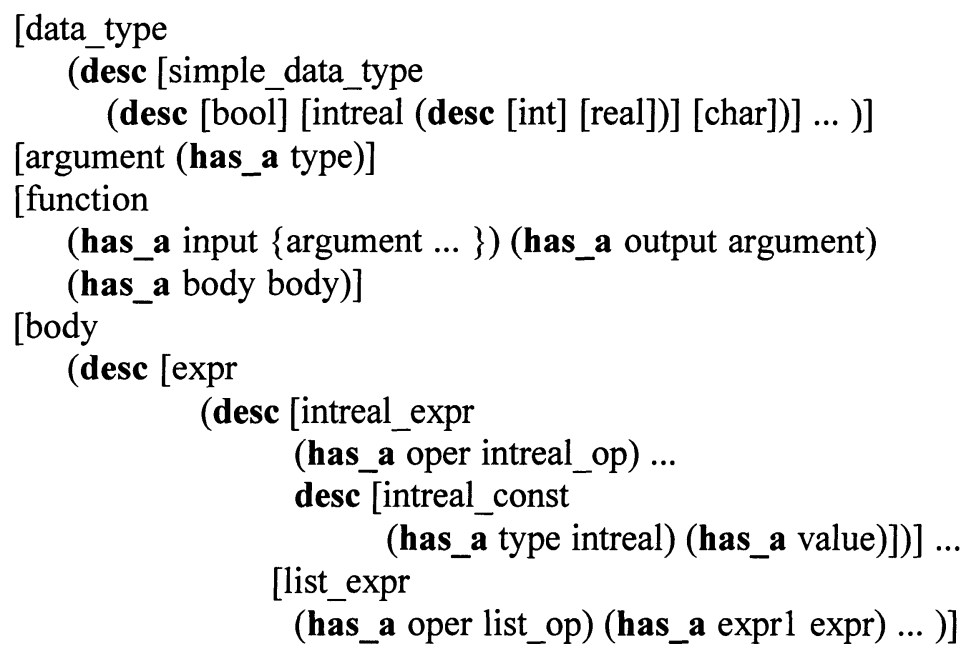

A taxonomy for the procedural programming language looks as follows.

[data_type

(desc [simple_data_type

(desc [bool] [intreal (desc [int] [real])] [char] )] ...

[reference (has_a type data_type)] )]

[argument (has_a type)]

[procedure

(has_a input $\{$ argument ... $\}$ ) (has_a output $\{$ argument ... $\}$ )

[expr

(has_a body instruction)]

(desc [intreal_expr

(has_a oper intreal_op) (has_a expr1 intreal_expr)... )] [instruction

(desc ...

[if (has_a cond bool_expr) (has_a then instruction)

(has_a else instruction)] ] ] ... )]

The main features used in this description desc, is_a and has_a create two

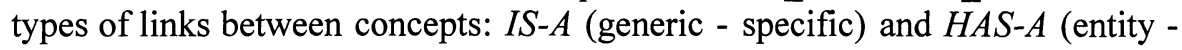
subentity).

To enable a student to write programs the basic domain knowledge must be augmented with samples of programs relevant for the types of common problems. As illustrating example a basic program operating on sets is considered. The data type which can be used for set modelling is the array or the user defined type list in the procedural language, and the list in the 
functional language. The function member, testing the set membership of an element, might in a procedural language have the form:

procedure member (in $\mathrm{x}$ : int; a: ^int; out r: bool)

var $p: \wedge$ int;

begin $\mathrm{p}:=\mathrm{a} ; \mathrm{r}:=$ false;

while $\mathrm{p} \diamond$ nil do

begin $r:=r$ or $p^{\wedge} \cdot n=x ; p:=p^{\wedge}$.next;

end;

end;

And in a functional language:

dec member: int, [int], bool

def member $x$ nil $:=$ false

member $\mathrm{x} y$ : $\mathrm{ys}:=\mathrm{x}=\mathrm{y}$ or member $\mathrm{x}$ ys

The two programs are instances of the same abstract representation:

Input: $\mathrm{x}$ : type $\mathrm{T}$, s: set of type $\mathrm{T}$

Output: boolean

Process: if empty $s$ then false else $x=s 1$ or $\ldots$ or $x=s_{n}$, where $s=\{s 1$, $\ldots \mathrm{sn}\}$

In our model a program is seen as an instance of the general procedure or function concept. For instance the function 'member' would look as follows:

[member

(is_a function)

(input $\{[x$ (is_a argument) (type int) $]$

[1_member (is_a argument) (type [list (type int)])]\})

(output [member_out (is_a argument) (type bool)])

(body [if1

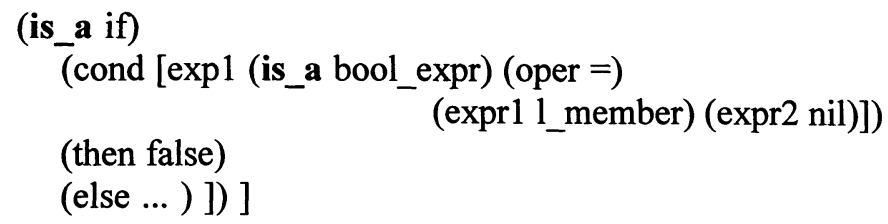

Although the programs are different, the main ideas such as the use of a list data type supporting the set implementation and the necessity to cover the whole set when performing the necessary tests, appear in both programs. 


\section{USE OF ANALOGY IN MODELLING KNOWLEDGE TRANSFER}

In the two conceptual hierarchies some similarities between specific concepts of the two paradigms can be outlined. For instance lists and arrays are similar data types deriving from a more general concept of homogenous composed data type; if structures which appear in both languages, are distinct embodiments of a more general structure. The appearance of repetition in the two languages is more complicated: the procedural language has a while structure to express repetition, while the functional one uses recursion for the same purpose.

Similarities between conceptual structures in the domain suggest thinking in analogies as a technique which can be used while modelling the programming process [10]. Two main problems have to be solved:

- modelling the knowledge transfer at the conceptual level through analogies between theoretical concepts [11];

- the effects which theoretical analogies have on the problem solving behaviour of the student.

We go back to our earlier example and compare this with a program which generates the sum of all elements of a list:

dec sum : [int], int

def sum nil $:=0$

sum $1:$ ls : $=1+$ sum $1 \mathrm{~s}$

A similarity analysis of the two programs through the representation

(member sum \{ ( $\mathrm{x}-)(\mathrm{y} l)$ (ys ls) (member_out sum_out)

(false 0$)(\mathrm{x}=\mathrm{y}$ l) $($ or +$)$

((member x ys) (sum ls)) \} )

shows correspondence between the main parts of these programs. The inconvenience of this representation is the lack of information concerning the degree of similarity [10]. The similarity of $y$ and 1 , for instance, is higher than that between member_out and sum_out which have different data types. The similarity of arguments is transmitted to the expressions from the function body. Some complications are raised by the application of thinking by analogy to programming. One is establishing the similarity of two programs solving the same problem which is easier to do at the formal level than at the semantic level. A solution might be the specification of preconditions and postconditions. This implies a complex specification language; and also preconditions and postconditions are not always known. Another issue is the adaptation step. The solution to a given problem cannot be structurally adapted, 
because this would imply determination of exact points in which the two solutions differ. As human analogy does not act as a mechanical translation or formal compilation, a model of thinking by analogy applied to programming must also consider derivational adaptation. The solution of a given problem cannot be obtained from one example only, the useful parts of several examples must be combined into a final solution.

\section{INSTRUMENT USED TO STUDY KNOWLEDGE TRANSFER}

An instrument to study the knowledge transfer which takes place when passing from one programming language to the next one, has been implemented based on the considerations presented above. Our instrument is an experimental prototype allowing to predict the behaviour of students translating a program from one programming language into the other. It has two main purposes:

- to allow the application of thinking by analogy in programming for problem solving;

- to support experiments for determining the influence of theoretical notions and examples shown on the performance of the problem solving process.

Our main goal is not the creation of a translation program, but the implementation of a model for student behaviour. At this stage the analogy between two programs can be established at the formal level. To solve some of the problems presented above we developed an algorithm for the determination of the similarity of two entities. The algorithm has two basic aims: to evaluate the similarity of two entities and to create a description of their similar aspects. The similarity evaluation will be used while deciding which is the solved problem (or problems) which will be used in the adaptation process. A similarity degree between 0 and 1 is calculated; for a value close to 1 the entities are very similar. The description of similar aspects is used in adaptation.

The main idea is to evaluate the degree of similarity of two entities by taking into account both the degree of similarity between their features and the importance of these features in the similarity determination process. Our algorithm has the following steps:

1. Initialization of cij which values describe the importance of feature $j$ while evaluating the similarity degree of two objects derived from the same abstraction $i$.

2. Initialization of matching context: $\boldsymbol{C M}=\boldsymbol{C M I}$ where $\boldsymbol{C M I}$ contains suppositions about initial matching.

3. Initialization of the set $\boldsymbol{E}$ of entities compared to the object $o$ considered.

4. Initialization of the set of already analyzed entities $\boldsymbol{C A}=\varnothing$. 
5. For each entity $e \varepsilon E$

5.1. $\boldsymbol{C A}=\boldsymbol{C A} \cup\{(\mathrm{e}, \mathrm{o})\} \mathrm{g}_{\mathrm{eo}}=0$.

5.2. For each pair of subentities $\left(\mathrm{se}_{\mathrm{j}}, \mathrm{so}_{\mathrm{j}}\right), \mathrm{se}_{\mathrm{j}} \varepsilon \mathrm{e}, \mathrm{so}_{\mathrm{j}} \varepsilon \mathrm{o}$

5.2.1. $g_{j}$ (the similarity degree associated to the pair) is calculated using values already included in $\boldsymbol{C A}$ and $\boldsymbol{C M}$.

5.2.2. $C M=C M \cup\left\{\left(\mathrm{se}_{\mathrm{j}}, \mathrm{sO}_{\mathrm{j}}, \mathrm{g}_{\mathrm{j}}\right)\right\}$.

5.3. $\boldsymbol{C A}=\boldsymbol{C A}-\{(\mathrm{e}, \mathrm{o})\}$

$\boldsymbol{C M}=\boldsymbol{C M} \cup\left\{\left(\mathrm{e}, \mathrm{o}, \mathrm{g}_{\mathrm{eo}}\right)\right\}$, with $\mathrm{g}$ calculated in the context of the common abstraction of $e$ and $o$.

An initial matching context $\mathrm{CMI}=\{$ (int bool 0.2$)\}$ sets a predefined value for the similarity of two data types, a value which will also be reflected through similarities of predefined operators and constants for the two types. We must also specify the values $c_{i j}$ for the elements occurring in the two programs:

(if (cond 0.4) (then 0.3) (else 0.3))

(list (type 1))

(operation (input 0.4) (output 0.6))

(expr (oper 0.4) (expr1 0.3) (expr2 0.3))

These values set the importance of each feature of an entity in the process of determining its similarity with another entity. For instance, the values $c_{i j}$ for an if structure are 0.4 for the condition and 0.3 for then and else branches. The analogy analysis must begin with the prototype of the function:

(x - 0) (1_member 1_sum 1) (member_out sum_out 0.2)

Values already calculated for argument similarity are again used in the algorithm:

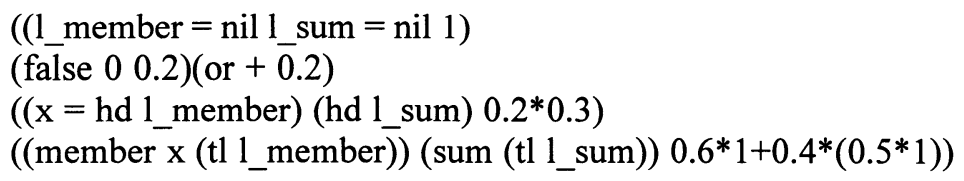

The similarity degree of the two bodies is $0.4 * 1+0.3 * 0.2+0.3 * 0.338=0.5614$. The result provided by the program in the 'member sum' example presented in the previous paragraph considering both the prototype and the body of the function is 0.43456 . The result also includes all intermediate pairs and values presented above; these will be used in the next step, the adaptation of the old solution.

An adaptation step performs both structural and derivational adaptation to obtain a new solution. Structural adaptation is used for simple items such as data types or variables, while derivational adaptation generates the body of the new function or procedure. 
While accomplishing program translation tasks, different failures might occur. The reason for a failure can lie in either similarity determination or adaptation. In both cases the failure must be repaired. The repair process must be followed by learning to retain the new experience. Learning is an incremental process [11] and implies two actions: acquisition of new knowledge from outside the system and reshaping of existing knowledge.

Knowledge acquisition is performed to assimilate theoretical notions and training examples. The process can be triggered at any time when present knowledge is not enough for solving a new problem. But the most important aspect of learning is knowledge reshaping. Indexes can be used to model the links formed between knowledge packages. Learning implies modification of these indexes according to new knowledge. Learning might also imply modification of the importance assigned to each feature of an entity in similarity degree through modification of $c_{i j}$. The main problem which occurs is the potential necessity to consider different combinations of $c_{i j}$ for different problems. This might lead to the existence of a matching context stored with each sample of a solved problem. Another problem is the empirical character of value selection which makes the task of modification very difficult.

\section{EXPERIMENTAL RESULTS}

An adequate representation of the ideas stated above is a case based reasoning system with as advantages [12] such as the capacity of modifying the system's behaviour according to past experience, the multitude of links in the knowledge base and the analogy based retrieval process. The basic structure of knowledge memory is the memory organization package [12].

The memory includes both conceptual knowledge and samples of solved problems which make up the cases of the system. A case includes two equivalent programs written in two programming languages and the transformations which lead from one program to the other. If derivational adaptation is required, the rules applied for solution generation and the arguments these are applied to must be specified.

A first version of the instrument shows a satisfactory problem solving performance. The use of 4-5 significant training examples allows the system to solve common problems which require calculus, iteration and set operations. The main examples we considered were a simple calculus problem, the member case presented above, the union of two sets and the factorial function.

The solutions for new problems provided by the model are similar from what we would expect from a second year student. The errors occurring during the problem solving process still have to be addressed. An adequate 
student model should provide similar errors to those usually made by students allowing diagnosis of their causes and improvement of the teaching process.

\section{CONCLUSIONS}

Quite a few cognitive psychologists and human factor researchers believe that humans use mental models to interact with devices. It therefore seems a good idea to qualitatively measure the kind of mental model which would be appropriate from both cognitive and computational perspectives. The learning process implies reshaping and usage of previous knowledge. When moving from one programming language to another, the basic problem solving ideas are transferred to the constructions of the new language. The knowledge transfer is performed both at the conceptual and at the behavioural level. The application of ideas coming from solved problems to new ones can be modelled by reasoning by analogy.

A prototype for the study of the influence of previous knowledge on the problem solving performance can be useful in suggesting the areas where improvements in the teaching process might occur. The problem solving performance does not depend on the quantity of knowledge supplied to the system, but on the analogies between concepts and on the quality of the solved problems supplied to the system.

The explicit representation of the knowledge needed in manipulating programs in two different programming languages and of the knowledge employed in improving the mental model of the learner seems to be very beneficial for the process of instruction. It helps the teacher/tutor during assimilation by the learner of the second programming language. However, a larger taxonomy would be required in practice than the one sketched in the present paper. Other aspects, like multiple mental models, should also be tackled for actual applications.

\section{REFERENCES}

1. Self, J. A. (1988) Artificial Intelligence and Human Learning. Chapman and Hall Computing.

2. Bourdeau, J. and Borne, I. (1991) Knowledge-based user interfaces to facilitate learning. PEG 91, Rapallo, pp. 1-11. 
3. Nwana, H.S. (1991) User modelling and user adapted interaction in an intelligent tutoring system. User-Modeling and User-Adapted Interaction, 1, pp. $1-32$.

4. Staggers, N. and Norcio, A. F. (1993) Mental models: concepts for human-computer interaction research. International Journal of Man-Machine Studies, 38, pp. 587-605.

5. Ikeda, M., Kono, Y. and Mizoguchi, R. (1993) Nonmonotonic model inference: a formalization of student modeling. Proceedings of 13th IJCAI, Chambery, pp. 467-473.

6. Koenemann, J. and Robertson, S. P. (1991) Expert problem solving strategies for program comprehension. Proceedings of CHI'91, New Orleans, Louisiana, pp. 125-130.

7. Kono, Y., Ikeda, M. and Mizoguchi, R. (1994) An inductive student modeling method which deals with student contradictions. IEICE Transactions on Information and Systems, pp. 39-48.

8. Ormerod, T. (1990) Human cognition and programming, in Psychology of Programming, (eds.) Hoc, J.- M., Green, T. R. G., Samurcay, R and Gilmore, D.

9. Porter, B., Bareiss, R. and Holte, R. (1990) Concept learning and heuristic classification in weak-theory domains. Artificial Intelligence, 45, pp. 229-263.

10. Falkenhainer, B., Forbus, K.D. and Gentner, D. (1989/90) The structuremapping engine; algorithm and examples. Artificial Intelligence, 41, pp. 1-63.

11. Letia, I.A. (1993) Transfer of conceptual knowledge in program comprehension. Proceedings of 7th International PEG Conference, Edinburgh, Vol. 1, pp. 366- 374.

12 Schank, R. C. and Riesbeck, C. K. (1988) Inside Case-Based Reasoning. Laurence Erlbaum Associates Publishers, Hillsdale, New Jersey. 